\title{
Simulation Model Driven Engineering for Manufacturing Cell
}

\author{
Hironori Hibino $^{1}$, Toshihiro Inukai ${ }^{2}$, and Yukishige Yoshida $^{2}$ \\ ${ }^{1}$ Technical Research Institute of JSPMI(Japan Society for the Promotion of Machine Industry), \\ 1-1-12 Hachiman-cho, Higashikurume, Tokyo, Japan \\ hibino@tri.jspmi.or.jp \\ ${ }^{2}$ DENSO Wave Incorporation, 1 Kusaki, Agui-cho, Chita, Aichi, Japan
}

\begin{abstract}
In our research, the simulation model driven engineering for manufacturing cell (SMDE-MC) is proposed. The purposes of SMDE-MC are to support the manufacturing engineering processes based on the simulation model and to extend the range of control applications and simulation applications using the PC based control. SMDE-MC provides the simulation model which controls and monitors the manufacturing cell directly using PC based control in the manufacturing system execution phase. Then when the simulation model acts in response to its behaviors, the manufacturing system is controlled by synchronizing the simulation model behaviors. In the manufacturing system implementation phase, the simulation model is mixed and synchronized with real equipment, real controllers, and management applications under a condition where parts of equipment, control programs, and manufacturing management applications are not provided in a manufacturing system.
\end{abstract}

Keywords: Manufacturing Cell, Simulation, Model Driven, PC Based Control, network middleware, and Engineering Process.

\section{Introduction}

Recently, PC (personal computers) based control is slowly becoming easier to be examined and used in industries, as open control platforms are getting advancing such as development of open interfaces for equipment and development of middleware for manufacturing systems [1]. It is possible to control equipment in manufacturing cells with stability and flexibility using the PC based control. The industries expect to extend the range of control applications using the PC based control beyond the traditional control using PLC (programmable logic controller).

The other hand, the industries need to design and make new products for the market in rapid succession, as it is becoming harder to keep the high value of a product in the market as a long seller [2][3]. It is important to reduce the lead-time for manufacturing engineering processes from the manufacturing system design and implementation phase to the manufacturing system execution phase. One of the solutions to realize the requirements is the front-loading method, which finds problems in advance and solves the problems at an earlier phase in the manufacturing engineering processes while limiting wasteful periods to the minimum by reducing the number of times needed to 
go back and refine the design. The front-loading method using simulation technologies has attracted the attention of the industries. However as the purposes of the present manufacturing system simulation do not include evaluation of manufacturing system implementation by mixing and synchronizing simulation, real equipment, real controllers, and management applications, there are many limitations concerning simulation applications in the manufacturing engineering processes. Therefore it is necessary to extend the range of simulation applications in the manufacturing engineering processes.

In our research, the simulation model driven engineering for manufacturing cell (SMDE-MC) is proposed. The purposes of SMDE-MC are to support the manufacturing engineering processes based on the simulation model and to extend the range of control applications and simulation applications using the PC based control. SMDEMC provides the simulation model which controls and monitors the manufacturing cell directly using PC based control in the manufacturing system execution phase. Then when the simulation model acts in response to its behaviors, the manufacturing system is controlled by synchronizing the simulation model behaviors. In the manufacturing system implementation phase, the simulation model is mixed and synchronized with real equipment, real controllers, and management applications under a condition where parts of equipment, control programs, and manufacturing management applications are not provided in a manufacturing system.

In this paper, the environment of the simulation model driven engineering for manufacturing cell (E-SMDE-MC) is proposed. The necessary functions for E-SMDE-MC are defined and developed. E-SMDE-MC consists of our developed manufacturing model driven simulator (EMU), our developed soft wiring system, and the industrial network middleware which is one of the semi-standard middleware. The validation of E-SMDE-MC was carried out through a case study.

\section{Typical Manufacturing Engineering Process and Its Problems}

Based on our analysis for the typical manufacturing engineering processes, manufacturing systems are established through four phases [4]. Figure 1 shows the typical manufacturing engineering processes.

Phase 1: This phase is the planning phase to define fundamental manufacturing requirements such as target production volumes, location and so on.

Phase 2: This phase is the manufacturing system design phase to fix manufacturing specifications such as the numbers of equipment needed, layout, manufacturing management as with the Kanban system and so on.

Phase 3-1: This is a period prior to the manufacturing system implementation phase. In this period, engineers implement hardware such as special machines, transfer machines, and software such as ladder programs, robot programs, operation panels, and production control programs.

Phase 3-2: This is a later period of the manufacturing system implementation phase. In this period, in order to undertake the manufacturing system trial operations, engineers partially operate hardware execution and software execution and accurately evaluate their executions from the viewpoints of the manufacturing systems.

Phase 4: This phase is the actual manufacturing system execution phase. 
In phase 1 and phase 2, a manufacturing system simulator plays an important role in designing and evaluating manufacturing systems by using a virtual factory model [5][6][7][8]. In phase 3-1, engineers separately develop hardware such as robots and special machining devices, and software such as ladder programs and production control programs. Then they independently evaluate their developed hardware or software in their place. In phase 3-2, the developed hardware or software is gathered together and adjusted in a real plant. If the delivery of the hardware is late, it is not possible to evaluate the software. If problems occur in this phase, it is necessary to go back to the previous phases. The problems are usually fatal and sometimes cause a delay of production [4][9].

As the purposes of the present manufacturing system simulation do not include evaluation of manufacturing system implementation by mixing and synchronizing simulation, real equipment, real controllers, and management applications, there are many limitations concerning simulation applications in the manufacturing engineering processes [4][9][10]. This difficulty has hindered precise and rapid support of a manufacturing engineering process. Consequently the lead-time is not reduced. Therefore it is necessary to extend the range of simulation applications in the manufacturing engineering processes.

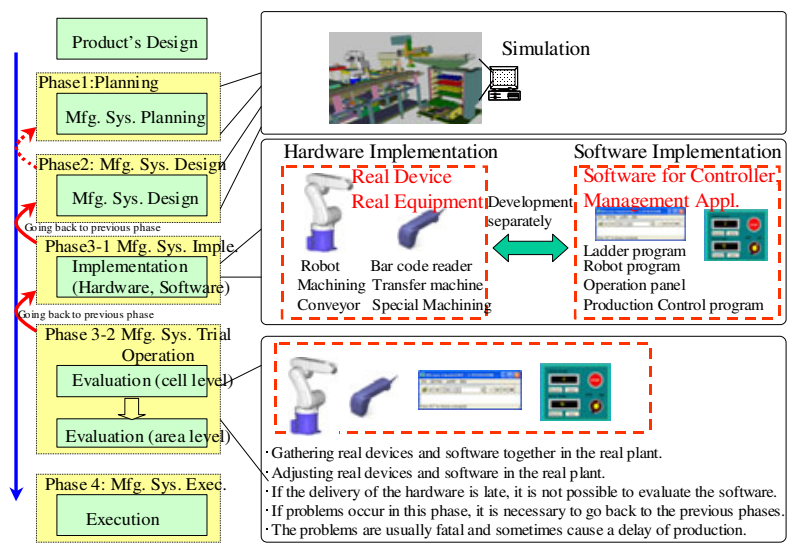

Fig. 1. Based on our analysis for the typical manufacturing engineering processes, manufacturing systems are established through four phases. This figure shows the typical manufacturing engineering process and its problems.

\section{Environment of Simulation Model Driven Engineering for Manufacturing Cell}

In our research, the simulation model driven engineering for manufacturing cell (SMDE-MC) is proposed. The purposes of SMDE-MC are to support the manufacturing engineering processes based on the simulation model and to extend the range of control applications and simulation applications using the PC based control. 
SMDE-MC provides the simulation model which controls and monitors the manufacturing cell directly using PC based control in the manufacturing system execution phase. Then when the simulation model acts in response to its behaviors, the manufacturing system is controlled by synchronizing the simulation model behaviors. In the manufacturing system implementation phase, the simulation model is mixed and synchronized with real equipment, real controllers, and management applications under a condition where parts of equipment, control programs, and manufacturing management applications are not provided in a manufacturing system. Figure 2 shows an outline of our proposed SMDE-MC.

To realize the environment of the simulation model driven engineering for manufacturing cell (E-SMDE-MC), the following functions are necessary.

1. A function to define simulation model behaviors in the simulation model.

2. A function to control real equipment in response to simulation model behaviors.

3. A function to monitor information from real world such as real equipment, and to drive the simulation model in response to the monitored information.

4. A function to display simulation model behaviors using three-dimensional animation.

To realize the first function for E-SMDE-MC, the following modeling methods are proposed.

1.1 A modeling method to define equipment motion behaviors programs using the tree structure.

1.2 A modeling method to define parallel processing programs.

1.3 A modeling method to define specification control programs using a script type programming language.

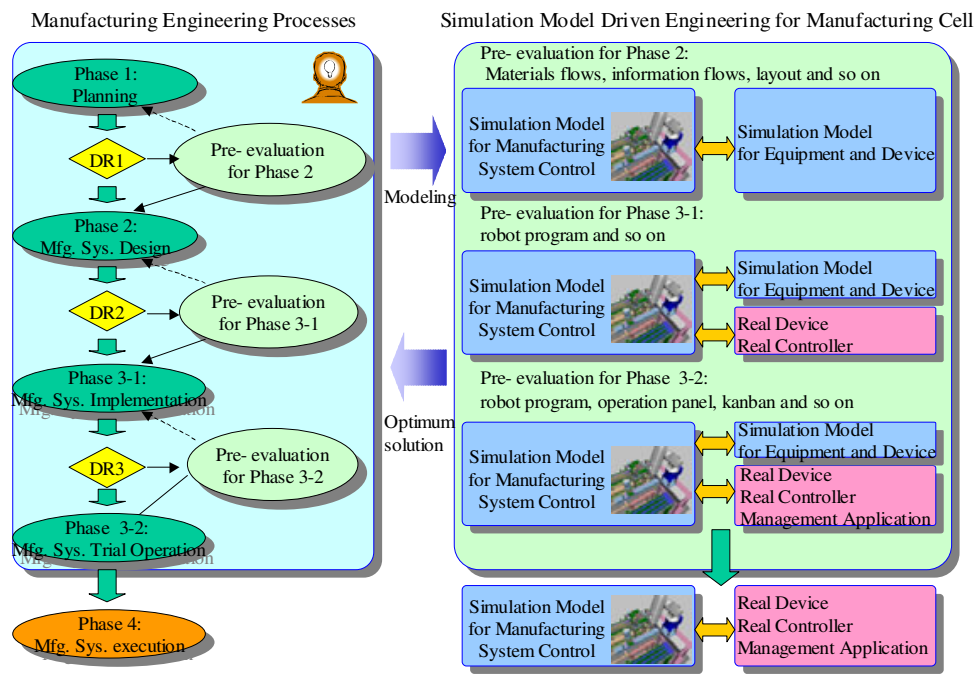

Fig. 2. This figure shows an outline of our proposed environment of the simulation model driven engineering for manufacturing cell (SMDE-MC) 
To realize the second function for E-SMDE-MC, the following mechanisms are proposed.

2.1 A mechanism to synchronize with several tasks such as control tasks for equipment and monitor tasks for equipment concurrently.

2.2 A mechanism to run specification control programs using a script type programming language.

2.3 A mechanism to synchronize simulation model behaviors in accordance with monitored information from real world such as robot state information, inspection equipment state information and so on.

To realize the third function for E-SMDE-MC, the soft wiring mechanism that has the following functions is proposed.

3.1 A wiring function to logically wire data on real world and data on the simulation model.

3.2 A transmission function to transmit signals and data between the simulation model and the real world.

To realize the forth function for E-SMDE-MC, the following function is proposed.

4.1 An animation function to visualize the three-dimensional simulation model by synchronizing the results of manufacturing cell behaviors.

In order to implement the first function, the second function, and fourth function, the manufacturing model driven simulator (EMU) is proposed and developed. In order to implement the third function, the soft wiring system is proposed and developed. ORiN (Open Resource interface for the Network) [11] is used as the semi-standard manufacturing middleware in E-SMDE-MC. The ORiN script language, which is provided as the standard script language on ORiN, is used to realize the 2.2 function. The application to interpret and execute the ORiN script language is used in E-SMDE-MC. Figure 3 shows a system structure of E-SMDE-MC. Figure 4 shows an outline of E-SMDE-MC. Figure 5 shows an outline of EMU.

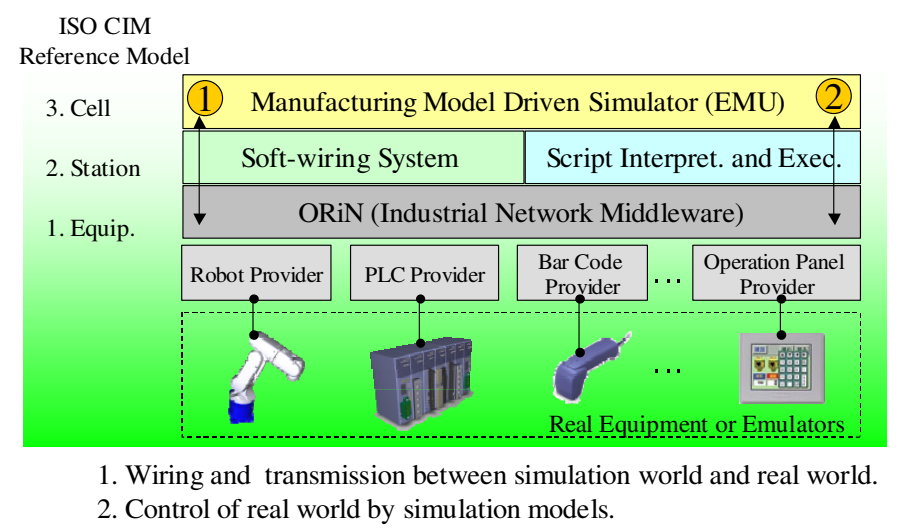

Fig. 3. This figure shows a system structure of our proposed environment of the simulation model driven engineering for manufacturing cell (E-SMDE-MC) 


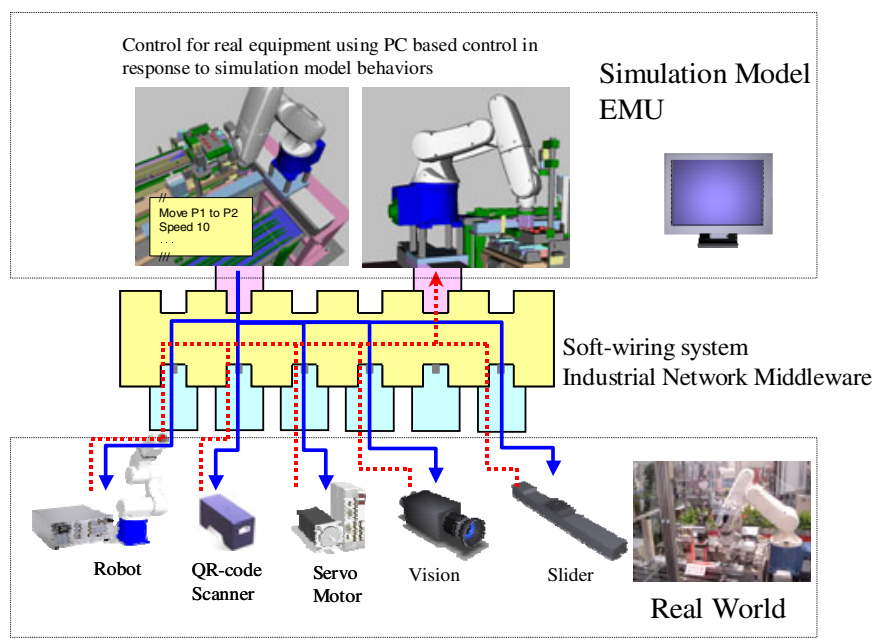

Fig. 4. This figure shows an outline of our proposed environment of the simulation model driven engineering for manufacturing cell (E-SMDE-MC)

\section{EMU}

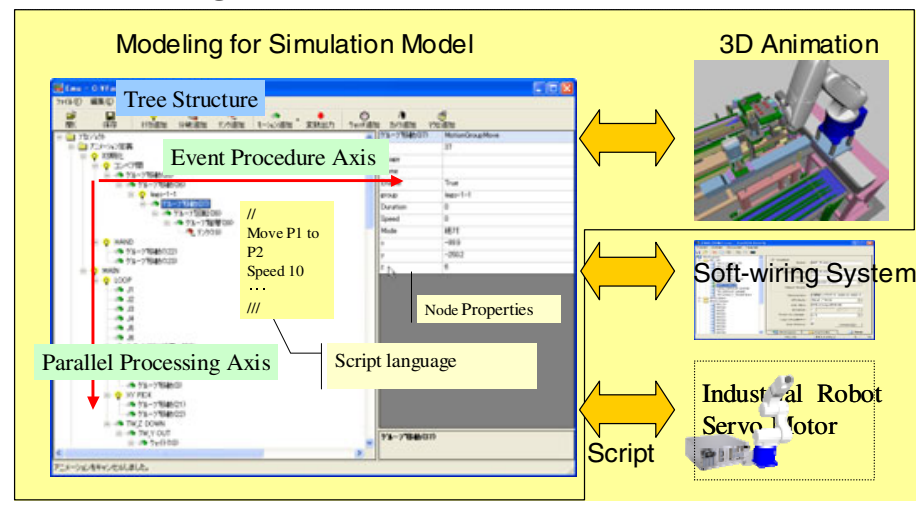

Fig. 5. This figure shows an outline of our proposed manufacturing model driven simulator (EMU) and its relationships with virtual model and real world

\section{A Case Study}

A case study was carried out using a small size of a manufacturing cell which consists of a robot, inspection equipment, vision equipment, servo motors, conveyors, a QR-code scanner and so on. It was confirmed that the simulation model for the case study manufacturing cell could be made on the manufacturing model driven simulator (EMU). It was also confirmed that the simulation model in EMU could control and monitor the manufacturing cell directly using PC based control. It was also confirmed that the simulation model in EMU could be synchronized with real equipment under a 
condition where parts of equipment such as the robot, inspection equipment, servo motors, conveyors and so on are not provided in the manufacturing cell. Figure 6 shows the case study manufacturing cell.

Through this case study, we confirmed that our proposed E-SMDE-MC could be used in the implementation and execution phase. E-SMDE-MC is a valid to support the manufacturing engineering processes based on the simulation model, and to extend the range of control applications and simulation applications using the PC based control.

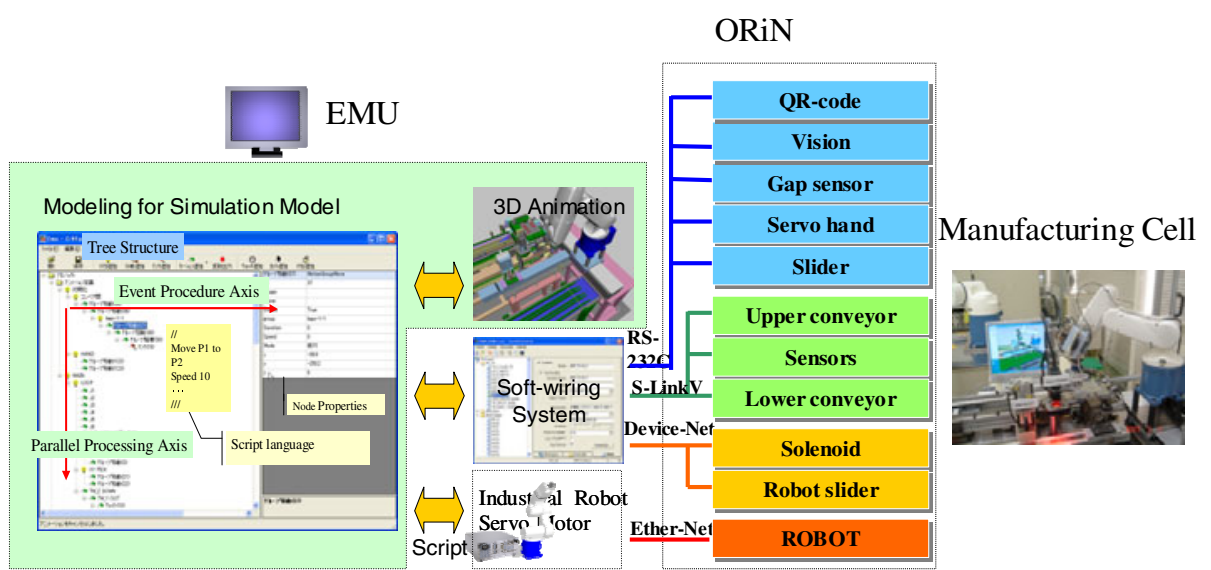

Fig. 6. A case study was carried out using a small size of a manufacturing cell which consists of a robot, inspection equipment, vision equipment, servo motors, conveyors, a QR-code scanner and so on. This figure shows the case study manufacturing cell and the simulation model on EMU.

\section{Conclusion}

In this paper, the environment of the simulation model driven engineering for manufacturing cell (E-SMDE-MC), which includes our developed manufacturing model driven simulator (EMU), our developed soft wiring system, and the industrial network middleware which is one of the semi-standard middleware, is proposed.

The results were:

1. To summarize the roles to extend the range of simulation applications based on our analysis for typical manufacturing engineering processes.

2. To propose E-SMDE-MC, which realizes to support the manufacturing engineering processes based on the simulation model and to extend the range of control applications and simulation applications using the PC based control.

3. To clarify necessary functions for E-SMDE-MC.

4. To confirm through a case study that the simulation model for the case study manufacturing cell could be made on the manufacturing model driven simulator (EMU) and the simulation model in EMU could control and monitor the manufacturing cell directly using PC based control. 
5. To confirm through a case study that the simulation model in EMU could be synchronized with real equipment under a condition where parts of equipment such as the robot, inspection equipment, servo motors, conveyors and so on are not provided in the manufacturing cell.

Acknowledgments. This research is a part of the research project on manufacturing support systems using industrial standard technologies in JSPMI. This project was supported by funding from JKA.

\section{References}

1. Hong, K., Choi, K., Kim, J., Lee, S.: A PC-based Open Robot Control System. Robotics and Computer-Integrated Manufacturing 17, 355-365 (2001)

2. Tanaka, K., Nakatsuka, N., Hibino, H., Fukuda, Y.: Module Structured Production System. In: 41st CIRP Conference on Manufacturing Systems, pp. 303-308. Springer, Heidelberg (2008)

3. Molina, A., Rodriguez, C., Ahuett, H., Cortes, J., Ramirez, M., Jimenez, G., Martinez, S.: Next-generation Manufacturing Systems: Key Research Issues in Developing and Integrating Reconfigurable and Intelligent Machines. I. J. Computer Integrated Manufacturing 18, 525-536 (2005)

4. Hibino, H., Fukuda, Y.: Emulation in Manufacturing Engineering Processes. In: 2008 Winter Simulation Conference, pp. 1785-1793 (2008), ISBN:978-1-4244-2708-6

5. Hibino, H., Fukuda, Y., Fujii, S., Kojima, F., Mitsuyuki, K., Yura, Y.: The Development of an Object-oriented Simulation System based on the Thought Process of the Manufacturing System Design. I. J. Production Economics 60, 343-351 (1999)

6. Hibino, H., Fukuda, Y.: A User Support System for Manufacturing System Design Using Distributed Simulation. Production Planning and Control 17, 128-142 (2006)

7. Hibino, H., Fukuda, Y., Yura, Y., Mitsuyuki, K., Kaneda, K.: Manufacturing Adapter of Distributed Simulation Systems Using HLA. In: 2002 Winter Simulation Conference, pp. 1099-1109. Institute of Electrical and Electronics Engineers, Inc., New Jersey (2002)

8. Hibino, H., Fukuda, Y.: A Synchronization Mechanism without Rollback Function for Distributed Manufacturing Simulation Systems. J. Japan Society of Mechanical Engineers 68, 2472-2478 (2002) (in Japanese)

9. Hibino, H., Inukai, T., Fukuda, Y.: Efficient Manufacturing System Implementation based on Combination between Real and Virtual Factory. I. J. Production Research 44, $3897-$ 3915 (2006)

10. Hibino, H.: Simulation Environment for Efficient Manufacturing System Design and Implementation Using Network Middleware ORiN and HLA. J. Society of Instrument and Control Engineers 46, 545-560 (2007) (in Japanese)

11. Inukai, T., Sakakibara, S.: Impact of Open FA System on Automobile Manufacturing. J. Automotive Engineers of Japan 58, 106-111 (2004) (in Japanese) 